Relations industrielles

Industrial Relations

\title{
Le Bill 25
}

\section{Jean-Réal Cardin}

Volume 22, numéro 2, 1967

URI : https://id.erudit.org/iderudit/027784ar

DOI : https://doi.org/10.7202/027784ar

Aller au sommaire du numéro

Éditeur(s)

Département des relations industrielles de l'Université Laval

ISSN

0034-379X (imprimé)

1703-8138 (numérique)

Découvrir la revue

Citer cet article

Cardin, J.-R. (1967). Le Bill 25. Relations industrielles / Industrial Relations, 22(2),

273-277. https://doi.org/10.7202/027784ar

Tous droits réservés (C) Département des relations industrielles de l'Université Laval, 1967
Ce document est protégé par la loi sur le droit d'auteur. L'utilisation des services d'Érudit (y compris la reproduction) est assujettie à sa politique d'utilisation que vous pouvez consulter en ligne.

https://apropos.erudit.org/fr/usagers/politique-dutilisation/ 


\section{COMMENTAIRES}

\section{LE BILL 25}

\section{Jean-Rgal Carden}

La * loi assurant le droit de l'enfant à l'éducation et instituant un nouveau régime de convention collective dans le secteur scolaire $\bowtie$, mieux connue sous le nom de Bill 25, aura fait couler beaucoup d'encre... et de salive.

C'est qu'il s'agit, en effet, d'une loi d'exception dont la portée est autrement plus grande que le but immédiatement visé, à savoir le retour en classe des enfants et des instituteurs en grève, ne saurait, à lui seul, sembler permettre. C'est une loi qui, non seulement, est venue mettre fin aux arrêts de travail dans le monde scolaire du Québec, mais qui en même temps vise à transformer dans ses structures mêmes, le régime des négociations collectives dans ce secteur. C’est une loi, enfin, qui déroge, au moins pour un certain laps de temps, aux prescriptions du Code du Travail adopté en septembre 1964 et auxquelles avaient été soumis les instituteurs et les commissions scolaires par l'insertion des dispositions du Bill 15 dans la dernière édition de ce Code en 1965.

Ce sont là les caractères fondamentaux de cette loi, ceux qui expliquent en profondeur les remous de protestation qui en ont accompagné la sanction chez les instituteurs, et dans le monde syndical en général, ainsi que la lutte farouche à laquelle s'est livrée l'opposition lors de sa discussion à l'Assemblée législative. Ce sont aussi ces mêmes caractères qui peuvent expliquer, selon nous, la perplexité qui semble être celle du grand public quant au jugement qu'il doit porter sur une telle loi.

Que contient, essentiellement, le Bill 25? D'abord, il ordonne aux instituteurs en grève de retourner au travail dans les quarante-huit heures de sa sanction. Peu de gens, croyons-nous, hors les instituteurs eux-mêmes et leurs supporteurs syndicaux, ont songé à contester le bien-fondé d'une telle disposition de la loi, étant donné l'état d'urgence qui commençait sérieusement à menacer la poursuite valable de l'année scolaire pour un nombre de plus en plus élevé d'élèves.

Tous, y compris les instituteurs en grève eux-mêmes, étaient conscients que les choses ne pouvaient se figer encore longtemps dans l'état où elles étaient, sans compromettre sérieusement l'année scolaire des élèves impliqués. Ce n'est pas la première fois au Canada où les pouvoirs publics mettent fin au moyen d'une loi spéciale, à un arrêt de travail qu'ils ont jugé préjudiciable à l'intérêt public. Les chemins de 
fer nous en ont donné un certain nombre d'exemples au cours des années récentes. Il appartient à l'autorité politique de prendre une telle décision là où, la grève, bien que permise, menace à un certain moment de nuire sérieusement à l'ensemble de la population ou à un secteur important de cette dernière.

Ce qui est particulier au Bill 25, c'est que dans le Québec, le Code du Travail prévoit déjà une telle situation d'urgence et qu'il contient précisément un mécanisme dont les pouvois publics peuvent se servir en de telles circonstances: c'est celui de l'article 99, prévoyant la constitution d'une commission d'enquête, l'émission d'une injonction et la suspension pour quatre-vingts jours, de la grève en cours. Il s'agit donc ici d'une suspension du droit de grève et de la grève elle-même, pour ce laps de temps, et non d'un retour définitif au travail, comme c'est le cas selon le Bill 25.

En dépit des défauts que nous pouvons reconnaitre à l'injonction de l'article 99 du Code et du ressentiment que ce seul vocable suscite chez l'ensemble du monde syndical, n'aurait-il pas été mieux d'appliquer les prescriptions du Code telles que le législateur les avait établies il n'y a pas encore trois ans?

Si le gouvernement ne l'a pas fait, c'est qu'il était conscient dle ces faiblesses inhérentes à la procédure de l'injonction, qu'il avait à l'esprit les quelques tentatives d'application qui en ont été faites depuis l'adoption du Code et de la Loi de la Fonction publique, et surtout, qu'il entendait prévenir une succession plus que probable de grèves régionales tout en se donnant le temps de mettre sur pied un nouveau mécanisme de négociation dans le secteur scolaire.

Nous touchons ici au deuxième objet du Bill 25, c'est-à-dire les dispositions qui visent à prolonger jusqu'au 30 juin 1968 les conventions collectives expirées avant le 11 février 1967 ainsi que celles encore en vigueur mais qui doivent expirer entre cette dernière date et le ler octobre de la même année.

Des implications, graves en elles-mêmes, découlent de ces disposition du Bill 25. Elles empêchent, jusqu'au 30 juin 1968, l'exercice légal du droit de grève par les associations d'instituteurs; elles fixent d'autorité les conditions salariales de ces derniers, pour la même période de temps; enfin, elles imposent l'arbitrage obligatoire là où des conflits peuvent survenir quant à l'application de la loi relativement aux paiements prévus en guise d'augmentation de traitements, quant à l'application des ententes intervenues avant le 11 février 1967 et permises par le Bill 25, ainsi que lors des négociations permises pour fin de revision des conventions prolongées, toujours évidemment, dans les limites prévues par le Bill.

Que penser de ces dispositions du Bill 25 qui mettent temporairement en veilleuse l'exercice du droit de grève récemment acquis par les 
instituteurs dans le Code du Travail, et qui limitent ainsi le droit à la libre négociation des conditions de Travail? En dépit de ce qu'en ont dit les instituteurs, le monde syndical, et la plupart des éditorialistes de nos quotidiens, d'une part, et les porte-parole gouvernementaux d'autre part, il n'est pas facile de porter un jugement sans nuance les approuvant ou les condamnant d'emblée et une fois pour toutes.

A première vue, elle nous apparaissent très dangereuses en ce qu'elles s'attaquent à des droits reconnus comme fondamentaux dans notre société économique, et qu'elles ne respectent pas les structures de négociations ni les mécanismes consacrés explicitement par le Code du Travail tout récemment adopté.

Si ces dispositions du Bill 25 sont le prélude à une réorientation de l'attitude gouvernementale susceptible de mener à des amendements de nature restrictive dans le Code du Travail, elles doivent être stigmatisées comme un précédent dangereux dans les services publics et dénoncées comme telles.

Mais peut-on faire un procès d'intention au gouvernement? Nous ne le croyons pas. Nous n'avons pas le droit de lui en faire un, croyonsnous, dans l'état actuel des choses. L'avenir seul nous dira si le Bill 25 est un premier pas dans la réaction ou s'il n'est qu'une mesure d'urgence n'entamant rien en longue période de la substance des droits acquis.

Bien sûr, peut-être les pouvoirs publics auraient-ils pu ordonner simplement le retour au travail, tout en permettant la continuation de la médiation, quitte à assouplir quelque peu les directives et à prévoir l'arbitrage obligatoire dans les cas où, malgré tout cela, des mésententes auraient subsisté. En principe, nous aurions de beaucoup préféré une telle attitude.

Cependant, il faut rappeler encore une fois que l'escalade des conflits localisés menaçait de s'étendre passablement, et que la confusion existante risquait de se prolonger longtemps si le système actuel de négociation était maintenu. Rien ne dit d'ailleurs que le climat des relations de travail dans ce secteur se serait amélioré pour la peine étant donné les attitudes respectives des parties en présence.

Il faut souligner ici d'ailleurs, que le gouvernement par les derniers amendements qu'il a apporté au Bill 25, a considérablement atténué dans les modalités de son application, les effets nocifs qui semblaient devoir découler de la mise en veilleuse des droits de négociation et de grève que cette loi proclame pour la période de temps qui y est fixée.

Tout en maintenant ses fameuses directives du 14 octobre 1966, il semble s'être efforcé de prévoir pour les instituteurs des conditions salariales qui nous semblent acceptables en soi; d'enlever l'arbitraire quant à la détermination des hausses prévues de traitements en pré- 
voyant l'arbitrage en cas de conflits possibles, à leur sujet, de laisser jouer les ententes intervenues avant le 11 février 1967 sur les objets autres que les traitements, et de permettre la négociation pour fin de révision des contrats prolongés, quant à ces mêmes objets, tout en prévoyant, évidemment, l'arbitrage obligatoire en cas de mésentente.

Ceci nous amène au troisième objet du Bill 25, lequel est d'instituer un nouveau régime de négociations collectives dans le secteur scolaire.

C'est en vue de l'établissement de ce nouveau régime, d'ailleurs, que le gouvernement a jugé bon de suspendre, dans la deuxième section du Bill, l'exercice des droits dont nous venons de faire état et la négociation telle qu'elle se pratiquait à venir jusqu'ici. On pourrait penser encore, comme l'opposition libérale le réclamait, qu'il aurait mieux vału dissocier l'étude de ce nouveau régime de négociation de la loi ordonnant le retour au travail des instituteurs.

A tout événement, c'est, selon nous, ce dernier aspect du Bill 25 qui est le plus important en même temps que le plus prometteur pour l'avenir des relations de travail dans le secteur scolaire. Nous référons ici, à l'idée même de la négociation à l'échelle du Québec qui est prévue au Bill. Il est devenu indispensable et urgent, pour qu'une véritable politique salariale dans le secteur scolaire puisse s'établir, que le laissezfaire collectif » actuel et le marchandage localisé, avec lanarchie qui les caractérise, fassent place à une négociation d'un niveau plus élevé, en l'occurence au niveau du Québec tout entier. C'est à ce niveau, et à ce niveau seulement, qu'un véritable statut de l'enseignant peut vraiment s'élaborer, que les ajustements les meilleurs peuvent survenir entre les disponibilités financières de la population québécoise et les conditions de travail et de traitement des instituteurs.

C'est sur une telle base, aussi, que la négociation collective peut le mieux, le plus sérieusement, et le plus rationnellement possible, prévoir et établir les différentiels éventuels de rémunération et de conditions de travail, afin de rendre justice aux différentes régions couvertes et aux divers groupes d'instituteurs qui y oeuvrent. La négociation à l'échelle du Québec ne signifie pas nécessairement l'uniformité absolue de son résultat, la convention collective, mais suppose au contraire, l'examen sérieux et rationnel des diversités de conditions nécessaires au fonctionnement optimum du marché du travail à travers l'ensemble du territoire. Le Bill 25 semble devoir permettre une telle politique de l'éducation. Il faut noter cependant que si des réformes considérables ne sont pas apportées aux plans de l'administration et du financement scolaire, les fruits de la négociation élargie risquent de ne pas passer la promesse des fleurs! De plus, le Bill 25 , bien que prévoyant un comité conjoint des administrations scolaires actuelles et des associations d'enseignants, auxquelles pourront s'ajouter deux représentants du ministère de l'Educa- 
tion, ne dit encore rien d'une éventuelle présence de l'Etat (présence directe) aux négociations d'envergure provinciale.

Il semble évident que le gouvernement ne pourra faire autrement que participer directement à ces négociations; il nous semble, en tout cas, impensable que seules, encore une fois, les administrations scolaires actuelles, par leurs associations, siègent du côté patronal. L'Etat est dorénavant le véritable employeur en éducation, et c'est avec lui que les instituteurs doivent discuter de leurs conditions de travail et d'emploi. L'ambiguité du " payant absent " qui a tenté d'intervenir indirectement tout en maintenant un système juridique désuet de négociation a assez duré. Que les instituteurs sachent à qui ils ont affaire une fois pour toutes.

Une dernière critique que l'on peut faire au Bill 25 c'est que la détermination des objets devant faire partie de la négociation à l'échelle provinciale sera faite par arrêté ministériel sur réception des recommandations du comité conjoint créé par le Bill. N'eut-il pas été mieux de permettre une discussion de cette importante matière par des organismes législatifs compétents avant de décider du régime final de telles négociations? De toutes façons l'avenir permettra, seul, de juger d'une façon plus complète des effets, bons ou mauvais, de cette loi.

\section{LE CODE DU TRAVAIL DU QUÉBEC (1965)}

Lo genèse du présent Code du travail (GERARD HEBERT). Le Droit d'association, s@n extension, ses limites (J.-R. CARDIN). La Commission des Relations de Travail (ANDRE ROY'). La Commission des Relations de Travail et l'accréditation syndicale (GERARD VAILLANCOURT). Le Code du travail et la convention collective (FERNAND MORIN). a procédure de négociation et le recours à la grève ou au locknut. (MARIUS BERGERON). Le règlement pacifique des conflits (ROBERT AUCLAIR). Points de vue sur le Nouveau Code du travail (ROGER THIBAUDEAU), (LOUIS LABERGE), (DENIS GERMAIN), (J.-P. DESPRES), (ANDRE DEOM), (LAVAL. GRONDINÉS), (RAYMOND PARENT), (LS-CLAUDE' TRUDEL). Le nouveau rôle du Ministère du travail (HON. CARRIER FORTIN). Code du Travail. Bill 55 - Loi de la fonction publique.

$$
\text { I volume, } 264 \text { pages - Prix: } \$ 4.00
$$

\section{LES PRESSES DE L'UNIVERSITÉ LAVAL}

